\title{
New Therapeutic Approaches in Treating Cancer
}

\section{Hemant KSY*, Abhay Raizaday and Susmitha Kasina}

Department of Pharmaceutics, JSS College of Pharmacy, JSS University

\section{Introduction}

After cardiovascular diseases, cancer is the second major cause of mortality in the western world accounting for $24 \%$ of all deaths. In European countries each year over three-quarters of a million people die from cancer [1]. Once cancer is diagnosed, a variety of possible treatment options can be considered.The choice of treatment depends on the type of cancer and the extent of its progress. Three basic strategies used in treatment of cancer are: surgery, radio-therapy and chemotherapy. These may be employed either alone or in combination with others [2].

Since the past two decades researchers are focusing on developing different strategies to treat cancer so that the treatment can be more effective. In this short communication we are focusing on the new approaches which have been in research for treating cancer. The approaches are described below:

\section{Inhibition of cancer cell metabolism}

Sometimes cancer cells enter a state known as senescence (programmed growth arrest). In this state, the tumour cells are inactive and no longer proliferate.

Dorr et al. [3] showed in his research work that when cancer cells enter senescence state they tend to increase energy metabolism and thecells truly crave for sugars. This is because the moment cells enter the state of senescence, they produce large quantities of protein messenger substances which are digested by cancer cells. The process of digestion is a high energy process and a block in this causes cell death. Hence inhibition of sugar metabolism causes cell death. The other targets that cause the cancer cell death are by blocking some metabolic enzymes like glucose transporter, hexokinase, pyruvate kinase M2, lactate dehydrogenase, pyruvate dehydrogenase kinase, fatty acid synthase etc. [3].

\section{Treating with monoclonal antibodies}

One approach to avoid side effects is to target specifically cancer cellsand nothealthy cells. In 1906, Paul Ehrlich coined the phrase "magic bullet" to describe a drug that specifically targets diseasecausing organisms. His magic bullet became a reality in the 1970s with the development of monoclonal antibodies ( $\mathrm{mAbs}$ ), which are designed to adhere onto specific targets found only in cancer cells. The first mAbs, Rituximab (Rituxan/MabThera) and Trastuzumab (Herceptin), were approved in the United States in the 1990s. Till date, $12 \mathrm{mAbs}$ have been approved for the treatment of cancer, the most recent being Brentuximabvedotin (Adcetris) for the treatment of lymphomas. They are highly effective in carefully chosen patientsand the side effect profile is better than cytotoxics [4].

\section{Protein accumulation in cancer cells leading to their death}

Ubiquitinated protein accumulation is a novel approach to treat cancer. Both the histone deacetylase (HDAC) inhibitor, Vorinostat and the HIV protease inhibitor, Ritonavir suppress the chaperone activity of heat shock protein (HSP) 90 and increase the amount of unfolded proteins in the cell. Combining either of them with the proteasome inhibitor, Bortezomib would kill cancer cells effectively by inhibiting the degradation of unfolded proteins and thereby causing ubiquitinated proteins to accumulate in the cells [5].

\section{Active immunotherapies}

Immunotherapies also known as cancer vaccines, stimulate a patient's immune system to destroy cancer cells. In 2010, the FDA approved first of such vaccines-Sipuleucel-T (Provenge) to treat advanced hormone-refractory prostate cancer. Vaccines trigger an immune response that may protect patients against metastasis and relapse. When compared to vaccines, immunotherapies exhibit lesser side effects but may cause inflammation at the site of action in few patients [6].

\section{Magnetic nanoparticles for cancer treatment}

Delivery systems can be harnessed to modify the release of systemic cancer agents, which allows them to remain longer in circulation and enables localization. These systems increase efficacy and minimize systemic exposure, thereby reducing side effects. Drug costs are also decreased by decreasing the amount of drug that has to be loaded. Linking magnetic nanoparticles to drugs will allow external magnets to pull the drug conjugate to the region of tumour. Thereby localizing the therapy and hence reducing side effects associated with the drug [7].

\section{Personalized medicine}

Gene expression diagnostics and biomarkers can be used to stratify patients into potential responders and non-responders or to select patients who are less likely to suffer side effects. This approach will help physicians to decide which regime is to be followed. Clinical trials also can be accelerated to ensure that non-responders are not exposed to unnecessary treatments. Genes specifically expressed in cancer cells, such as PEG-3 (Parentally Expressed Gene), can improve cancer imaging, thereby increasing the chance of early diagnosis.

\section{Treating cancer with anti-angiogenesis agents}

Formation of new blood vessels inside the tumour cell is called angiogenesis. New vessels created through this process are required for tumour growth and for metastases. But tumours use an organism's normal coagulation system to grow. This inturn leads to a number of coagulation disorders (coagulopathies) in cancer patients. By shutting down the angiogenesis process cancer cell do not grow and the anticancer agent can treat the cancer cells more effectively [8]. Transmembrane receptor tissue factor (TF) is a tumour determinant. It forms a complex with factor VIIa which helps in tumour angiogenesis. TF exists in two isoforms, alternatively spliced TF (asTF) and full length TF (flTF). flTF has effect on coagulation where asa sTF is said to reduce angiogenesis without effecting thrombogenicity $[9,10]$

*Corresponding author: Hemant KSY, Department of Pharmaceutics, JSS College of Pharmacy, JSS University, Mysore, Karnataka - 570015, India, Fax: 0821-2548359; E-mail: haisunny2@yahoo.co.in

Received August 12, 2014; Accepted September 19, 2014; Published September 22, 2014

Citation: Hemant KSY, Raizaday A, Kasina S (2014) New Therapeutic Approaches in Treating Cancer. Pharmaceut Reg Affairs 3: 128. doi:10.4172/21677689.1000128

Copyright: (c) 2014 Hemant KSY, et al. This is an open-access article distributed under the terms of the Creative Commons Attribution License, which permits unrestricted use, distribution, and reproduction in any medium, provided the original author and source are credited. 


\section{Oncolytic adenoviruses in treatment of cancer in humans}

After a century of volatile interest in treating tumours with viruses, adenovirus has been used increasingly in the past century to treat tumours.

Technical innovations have led to the generation of safer oncolytic viruses, which can also be armed to make the virus more potent. Currently, it seems that oncolysis alone is not able to cure advanced cancers due to intratumoural complexities. Thus utilization of the immunogenic effects of oncolysis for activating anticancer immunity seems useful. One approach to allow patients access to oncolytic virus therapies was the Advanced Therapy Access Program, operational between 2007 and 2011, whose lessons are now being implemented in standard clinical trials. Eventually, randomized blinded trials are needed to confirm efficacy, and several of them are ongoing with different oncolytic viruses [11].

\section{Conclusion}

Research for treating cancer has advanced rapidly in the past 70 years, from cytotoxic agents that shrank tumours but with significant systemic side effects to targeted therapies that can trigger specifically cancer cell death. Hence some of the most promising approaches have been described in this short communicationwhich are the current area of interest among the scientists throughout the world.

\section{References}

1. Cancer Research Campaign (1992). Cancer in the European community. Fact sheet, 5 .
2. Hoijatsadeghi-aliabadi, Amrollahahmadi (2003). Cytotoxicity and antitumour properties of a marine compound, hesa-aon cancer cells. DARU J Pharm Sci 11: 82-87.

3. Dörr JR, Yu Y, Milanovic M, Beuster G, Zasada C, et al. (2013) Synthetic lethal metabolic targeting of cellular senescence in cancer therapy. Nature 501: 421 425

4. Scott AM, Wolchok JD, Old LJ (2012) Antibody therapy of cancer. Nat Rev Cancer 12: 278-287.

5. Laemmli UK (1970) Cleavage of structural proteins during the assembly of the head of bacteriophage T4. Nature 227: 680-685.

6. Scott AM, Wolchok JD, Old LJ (2012) Antibody therapy of cancer. Nat Rev Cancer 12: 278-287.

7. Alexiou C, Tietze R, Schreiber E, Jurgons R, Richter H, et al. (2011). Cancer therapy with drug loaded magnetic nanoparticles-magnetic drug targeting. $J$ Magn Magn Mater 323: 1404-1407.

8. Mackey JR, Kerbel RS, Gelmon KA, McLeod DM, Chia SK, et al. (2012) Controlling angiogenesis in breast cancer: a systematic review of antiangiogenic trials. Cancer Treat Rev 38: 673-688.

9. van den Berg YW, Osanto S, Reitsma PH, Versteeg HH (2012) The relationship between tissue factor and cancer progression: insights from bench and bedside. Blood 119: 924-932.

10. Eisenreich A, Zakrzewicz A, Huber K, Thierbach H, Pepke W, et al. (2013) Regulation of pro-angiogenic tissue factor expression in hypoxia-induced human lung cancer cells. Oncol Rep 30: 462-470.

11. Pesonen S, Kangasniemi L, Hemminki A (2011) Oncolytic adenoviruses for the treatment of human cancer: focus on translational and clinical data. Mol Pharm 8: $12-28$ 